\title{
Pautas de crianza en el sector rural
}

Parenting guidelines in the rural sector

Diretrizes de parentalidade no setor rural

\author{
Asbleydi Bejarano \\ Corporación Universitaria Iberoamericana (Colombia) \\ Luz Alba-Beltrán \\ Corporación Universitaria Iberoamericana (Colombia) \\ Nancy Pineda \\ Corporación Universitaria Iberoamericana (Colombia)
}

D0l: https://doi.org/10.35622/j.rep.2021.02.004

Recibido 02/12/2020/ Aceptado 20/03/2021

RESUMEN. El presente artículo hace un análisis acerca de las experiencias en las pautas de crianza en los niños y niñas del sector rural, tema de gran relevancia a nivel nacional por sus condiciones sociodemográficas y psicosociales, enfocadas en algunas investigaciones relacionadas con el tema, reconociendo que las pautas de crianza cambian y se modifican de acuerdo con el desarrollo evolutivo, sociocultural, educativo, psicológico y afectivo de las familias, influyendo en el proceso de formación y construcción de la identidad de los menores a sus primeros años. Las familias son importantes para orientar valores, normas, principios, pautas en los infantes y que permitirá un adecuado y equilibrado desarrollo de la personalidad y una prevención de problemas de identidad, depresión, agresividad y baja autoestima.

PALABRAS CLAVE: Familia, pautas de crianza, experiencia, infancia, emoción.

ABSTRACT. The objective of this work was to determine the meaning of the poem "El dolor de la noche" by Jose María Eguren from the perspective of discourse analysis. This research corresponds to the qualitative approach and is descriptive. The method used to analyze the poem was hermeneutics and as a technique the analysis of poetic discourse that encompassed the textual, enunciative, semiotic and pragmatic dimension. As a result, the sadness and suffering caused by the loss of a loved one is evident, finding the subject in a state of loneliness, expressing messages of remorse and will go for what he lost and did not know how to live at the time.

KEYWORDS: Family, breeding guidelines, experience, childhood, emotion.

RESUMO. Este artigo faz uma análise sobre as vivências nos padrões de educação de crianças do setor rural, tema de grande relevância em âmbito nacional devido às suas condições sociodemográficas e psicossociais, com foco em algumas pesquisas relacionadas ao tema, reconhecendo que as Diretrizes da Maternidade mudam e se modificam de acordo com o desenvolvimento evolutivo, sociocultural, educacional, psicológico e afetivo das famílias, influenciando o processo de formação e construção da identidade dos menores nos primeiros anos. A família é importante para orientar valores, normas, princípios, orientações na criança e que permitirá um desenvolvimento adequado e equilibrado da personalidade e prevenção de problemas de identidade, depressão, agressividade e baixa autoestima. 
PALABRAS CLAVE: Família, padrões parentais, experiência, infância, emoção.

\section{INTRODUCCIÓN}

Las familias son protagonistas en el desarrollo socioafectivo de los niños y niñas, ya que son el primer centro de socialización infantil y juvenil, un campo para la adquisición de valores, normas, roles y habilidades que se aprenden durante este periodo; influenciadas por factores socioeconómicos, culturales, demográficos y educativos. Aunque son diversos los factores que afectan el desarrollo de los niños y niñas, es necesario reconocer la importancia del núcleo familiar en los estilos y pautas de crianza en el desarrollo socioafectivo y construcción de la identidad, previniendo así, trastornos en la formación de su personalidad.

Tenemos a Baumrind (1967, 1971), para quien las pautas de crianza deben ser flexibles atendiendo a las necesidades de sus hijos en la parte emocional, física y sexual de acuerdo con cada etapa de desarrollo. Los padres deben mantener el equilibrio del carácter y autoridad que permita la construcción de valores, principios y normas básicas para la generación de una personalidad bien fundamentada. Solís-Camara et al. (2007) definió la crianza como las actitudes y comportamientos de los padres y también realizaron investigaciones para establecer los factores que afectan la participación de los padres, identificando el bienestar subjetivo, las actitudes y las expectativas sobre el desarrollo del niño.

Se puede desprender que la familia como unidad para la convivencia, el bienestar, el apoyo y el logro de objetivos comunes desde esta perspectiva la familia se constituye como un grupo de personas que conviven, comparten vínculos afectivos y valores en la búsqueda de estados de bienestar y logro de objetivos comunes, además de satisfacer necesidades afectivas de compañía y apoyo. Los padres cumplen la función de desarrollar y promocionar pautas de crianza, vínculos de apego y patrones de éxito de manera integral. Las mayores preocupaciones giran en torno a la economía y hacen de las etapas del desarrollo y/o los aspectos de carácter y personalidad de sus hijos una oportunidad para la promoción del desarrollo y la integración familiar,

Otras teorías mencionan que la familia como estructura básica de la sociedad, tiene como finalidad a la educación y la formación para el desarrollo personal y colectivo a través de la transmisión de valores y pautas educativas que contribuyen al desarrollo social. La promoción de normas y límites, así como de patrones de éxito claro y definido como la escolaridad son las principales tareas de los padres. Se conciben el afecto y la confianza, parte de los vínculos de apego como el principal reto y complicación en estas familias.

Por otro lado, se tiene a familia como entidad ideal, esta se describe con adjetivos ideales y románticos que pueden llegar incluso a situaciones utópicas, su formación se refiere al logro de una realización personal para el cumplimiento de metas definidas idealmente, los vínculos 
de apego cálido y cercano son características de los padres y parte fundamental de la convivencia. La adolescencia y la juventud resulta un reto para la mayor parte de los padres que sostienen esta teoría.

Por último, se tiene a la familia como un conjunto de personas que comparte principalmente lazos sanguíneos y de parentesco. La familia se concibe como un conjunto de personas que comparten lazos sanguíneos y de parentesco con funciones diferentes en relación con la cercanía o lejanía de los mismos lazos, su principal función es la preservación de la especie y su desarrollo evolutivo. El establecimiento de normas y límites es la principal función educativa de los padres, lo que a su vez se considera como la parte medular y difícil de la crianza.

Según Erikson (1982), el desarrollo psicosocial continúa durante toda la vida y que en todas las etapas se presentarán crisis para poder pasar a la siguiente etapa. De acuerdo con Erikson, la etapa de laboriosidad, en la cual la interacción social es un factor determinante para el desarrollo integral de los individuos, así como para su personalidad, esta interacción social tiene extremos tanto positivos como negativos, donde se genera un sentido de iniciativa contra sentimientos de inferioridad, todo lo anterior incidiendo en el desarrollo de la personalidad. El desarrollo de la personalidad de acuerdo con las teorías dinámicas de (Freud, Erikson y White) y su aprendizaje social, se puede decir que la incorporación o influencia de los atributos de otros aporta patrones para construir su propia personalidad.

La idea de investigación toma como referente a Erikson quien señala que, en cada una de sus etapas y desarrollo, los seres humanos deben pasar por un proceso desde la infancia hasta edad adulta tardía, donde cada ser se enfrenta a situaciones y experiencias, las cuales permitirán que domine y genere nuevos retos en su vida futura.

De esta manera, analizaremos como la familia se convierte en el eje central de la educación de los infantes, desde el sector rural en el municipio de Gachalá, donde intervienen muchos factores, tanto económicos, como sociales, culturales, tecnológicos, ambientales y elementos de arraigo desde cada una de las familias, que hacen que las pautas de crianza en los hogares, sean distintas, pero que se enmarcan dentro del campo de la enseñanza, vital para la adquisición de valores, capacidades y habilidades propios para que el infante de sus primeros pasos hacia la exploración de la sociedad, donde los padres buscan madurar sus saberes y percepciones para que lograr acoplarse con solidez a este nuevo espacio que va encontrando en el paso del tiempo.

\section{MÉTODO}

La metodología se basa en la investigación cualitativa ya que se pretende comprender la calidad de un hecho social en cuanto a construcción social para apoyar su transformación, y por lo tanto, su interés se centra en los significados singulares que le ha otorgado dicha comunidad, además de ser la opción más pertinente para indagar sobre un tema tan sensible como son las pautas de crianza al interior de las familias rurales y sus 
consecuencias para dar una visión más holística sin salir del contexto del cual se está trabajando.

En la investigación se desarrolló, desde la fenomenología, la cual pretende explorar las experiencias que las personas asignan a sus vivencias, ya sea mediante la descripción fiel de sus narraciones o mediante las interpretaciones que el propio investigador realiza de ellas; la fenomenología se preocupa más por los aspectos esenciales de la experiencia vivida.

Se utilizó la entrevista a profundidad como técnica de recolección de datos, las cual tiene como funcionalidad identificar las experiencias de pautas de crianza en los niños del sector rural. La muestra para esta investigación fueron 10 niños entre las edades de los 7 a los 10 años, los cuales cumplieron con características poblacionales como nivel socio económico, educativo, social y cultural, dentro de la muestra 6 eran mujeres y 4 hombres.

Para el desarrollo de la investigación se tuvo en cuenta algunos aspectos éticos, abordados en la resolución 008430 de 1993 del Ministerio de Salud. En el capítulo 1, artículo 5, menciona el criterio de mantener el respeto a su dignidad y la protección de sus derechos y su bienestar durante el proceso investigativo, igualmente se toma el literal (e) del artículo 6, donde se contará con el consentimiento informado y por escrito de los sujetos de investigación o su representante legal con las excepciones dispuestas en la resolución mencionada anteriormente. Asimismo el artículo 8, señala la importancia de proteger la privacidad del individuo, sujeto de investigación, identificándolo solo cuando los resultados lo requieran y éste lo autorice, además el artículo 16, tiene en cuenta el consentimiento informado, del sujeto pasivo de la investigación, para que sea válido debe cumplir con los requisitos establecidos en este mismo artículo, en cuanto al nivel comunicativo, los niños y padres fueron consultados para determinar así su interés en la investigación.

\section{RESULTADOS Y DISCUSIÓN}

De acuerdo con las trascripciones de las entrevistas se identificaron 25 códigos que se refieren a términos relevantes para nuestra investigación, los cuales se han agrupado en siete familias de códigos siendo analizados y de los cuales se obtuvo información sobre la experiencia de pautas de crianza en los niños de las familias rurales. Estas entrevistas permitieron reconocer las prácticas frente a las pautas de crianza que experimentan las familias rurales del municipio de Gachalá.

Las familias rurales presentan de acuerdo con su nivel socioeconómico, educativo y geográfico características en cuanto a su modo de vida y de orientar pautas, normas y principios en la formación de sus hijos. En el sector rural se presenta con mayor frecuencia la disfuncionalidad en los hogares, lo cual afecta la parte socioafectiva de los menores.

La educación que reciben los niños en los centros educativos juega un papel importante en la orientación e información sobre temas básicos en cuanto a la construcción de la identidad, 
a los cambios físicos y psicológicos de acuerdo a su edad, reconociendo características de culturas diferentes a la propia, ya que en la mayoría de los hogares es mínima la información que reciben sobre estos temas.

Otro aspecto que se evidenció, en esta muestra es que debido a las mismas tradiciones familiares y a las actividades propias del sector rural hay poco espacio para que los niños desarrollen actividades recreativas que les permitan socializar y disfrutar de acuerdo a su etapa de desarrollo, y por el contrario, manifiestan que deben realizar oficios como (encerrar los terneros, recoger leña entre otros).

Esta investigación se orientó con el objetivo de comprender las experiencias en las pautas de crianza de los niños del sector rural del municipio de Gachalá y de acuerdo a los resultados de la codificación podemos rescatar que el ambiente familiar en el contexto rural posee características particulares como la tranquilidad, factores ambientales más favorables y menos contaminantes que aportan en cierta medida al bienestar de sus habitantes, pero a su vez existe un cierto desconocimiento de condiciones que puedan aportar al desarrollo de conductas fundamentales para la orientación y desarrollo integral de cada uno de los miembros de las familias.

Los hallazgos de la presente investigación indican que las familias del sector rural en relación con la teoría sobre familia, no hay claridad en cuanto al concepto, dimensión e importancia de la función de esta, en el desarrollo integral de cada uno de sus miembros y por ende esto se ve reflejado en el sentir y pensar de los niños, los cuales expresan de cierta manera una insatisfacción afectiva y de bienestar en su hogar.

Según Según Baumrind $(1967,1971)$, las pautas de crianza deben ser flexibles atendiendo a las necesidades de sus hijos en la parte emocional, física y sexual de acuerdo con cada etapa de desarrollo. Los padres deben mantener el equilibrio del carácter y autoridad que permita la construcción de valores, principios y normas básicas para la generación de una personalidad bien fundamentada, lo cual no se relaciona con lo manifestado por los niños y niñas entrevistadas, ya que las orientaciones que reciben de su hogar no atienden a todas las necesidades que requieren las dimensiones del ser humano.

Se encontró también, que por las actividades económicas y agrícolas que se realizan en el sector rural, los padres generalmente se dedican a trabajar fuera de sus hogares los 7 días de la semana y por ende los menores en muchas ocasiones permanecen solos o al cuidado de un tercero, lo cual afecta de manera directa y pone en riesgo el bienestar, el desarrollo y la orientación de normas, valores, pautas y principios que fortalecen la construcción de la identidad basada en el aspecto afectivo y no solo en la satisfacción material.

En el sector rural la educación juega un papel importante, ya que los niños de acuerdo con las respuestas dadas en las entrevistas manifestaron recibir orientaciones y formación de parte de los docentes en cuanto a temas de sexualidad, desarrollo y manejo de emociones, información que no reciben por parte de algunos padres o cuidadores. 
Otro aspecto que se evidenció en esta experiencia y de acuerdo a las respuestas de algunos participantes, es su inconformidad frente a la mínima oportunidad que tienen para expresar, opinar o decidir sobre situaciones del mismo hogar y sobre su propio desarrollo (forma de vestir, elección de juego, de amigos). Esto debido a factores como bajo nivel educativo, económicos y social de los padres y a su vez la práctica de pautas que vienen de generación en generación dejando de lado los cambios en el desarrollo de sus hijos.

\section{CONCLUSIONES}

De acuerdo con las teorías que fundamentan esta investigación, se logró identificar un alto índice de desconocimiento que presentan la mayoría de los padres y cuidadores del sector rural del municipio de Gachalá, en cuanto a su rol como orientadores principales en el desarrollo de procesos afectivos, físicos intelectuales y sociales.

En vista de la realidad identificada en cuanto a pautas de crianza y apoyo de las familias en el proceso de desarrollo de sus hijos, es fundamental involucrar a las entidades públicas para la capacitación, formación y orientación de los hogares para ampliar su visión sobre la importancia de un ambiente familiar más afectivo y de mayor comunicación.

\section{REFERENCIAS}

Aguirre, E. (2000). Socialización y prácticas de crianza. En E. Aguirre y E. Durán.

Socialización: Prácticas de Crianza y cuidado de la salud. Bogotá, D. C.: CES. Universidad Nacional de Colombia.

Barahona, M. (2012). Familias, hogares, dinámica demográfica, vulnerabilidad y pobreza en Nicaragua, 7-59. Disponible en: http://www.eclac.org/publicaciones/ xml/3/26013/lcl2523-P.pdf

Beriain, J. (1990). Representaciones colectivas y proyecto de modernidad. Barcelona: Anthropos.

Berk, L. (2004). Awakening Children's Minds: How Parents and Teachers Can Make a Difference. Oxford University Press.

Cabrera, V., Guevara, I. \& Barrera, F. (2006). Relaciones maritales, relaciones paternas y su influencia en el ajuste psicológico de los hijos. Acta Colombiana de Psicología, 9 (2), 115-126.

Cole, P. M., Bruschi, C. J., \& Tamang, B. L. (2002). Cultural differences in children's emotional reactions to difficult situations. Child Development, 73(3), 983-996

Constitución política de Colombia Art, 44, Julio de 1991(Colombia).

Código de Infancia y adolescencia, Art 3, 10, 22, 23,39, Ley 1098 de 2006 (Colombia).

Clerici, Gonzalo; García, María Julia. Autoconcepto y Percepción de pautas de crianza en 
niños escolares. Aproximaciones teóricas, anuario de investigaciones, vol. XVII, 2010, pp. 205-212.Buenos Aires, Argentina.

Mansilla, M. (2000) Etapas del desarrollo humano Revista de Investigación en Psicología, Vol.3 No. 2.

Mendoza-Nápoles CA, Maldonado SEB. Revisión de las teorías y modelos incidentes en el desarrollo. Rev Elec Psic Izt. 2019; 22(1):1-24.

Myers, R. (1994). Prácticas de crianza. Bogotá: CELAM-UNICEF

Eisenberg, N., Fabes, R. A., Shepard, S. A., Ghrie, I. K., Murphy, B. C., \& Reiser,M. (1999).

Parental reactions to children's negative emotions: Longitudinal relations o quality of children's social functioning. Ld. Development, 70(2), 513-534.

Enciclopedia británica (2009). La familia, conceptos, tipos y evolución.

Consultado el 1 de mayo de 2014. Disponible en: http://cvonline.uaeh.edu.mx/Cursos/ BV/S0103/Unidad\%204/lec_42_LaFam_ConcTip\&Evo.pdf

Eraso, J, Bravo, Y \& Delgado, M. (2006). Creencias, actitudes y prácticas sobre crianza en madres cabeza de familia en Popayán: un estudio cualitativo. , 23-40.

Erikson, E. H. (1982). The life cycle completed. New York: Norton.

Fabes, R. A., Leonard, S. A., Kupanoff, K., \& Martin, C. L. (2001). Parental coping with children's negative emotions: Relations with children's emotional and social responding. Child Development, 72,907-920.

Freud, A., El yo v Im mecanismos de defensa, Paidós, Buenos Aires, 1954

Gallego,T.(2012)familias, infancias y crianza: tejiendo humanidad. Universidad católica del norte No35. Medellín. Retomado de http://wwwredalyc.org/pdf/1942/194224362005.pdf

Gligo, N. (2001). La dimensión ambiental en el desarrollo de América Latina. Santiago: Cepal.

Gómez, S. (2003). La nueva ruralidad: ¿qué tan nueva? Santiago: LOM.

Harris, P. L., Olthof, T., Meerum Terwogt, M., \& Hardman, C. (1987). Children's knowledge of situations that provoke emotion. International Journal of Behavioral Development, 10, 319-343.

Harter, S. (1993). Developmental changes in self-understanding across the 5 to 7 shift. In A. Sameroff \& M. Haith (Eds.), Reason and responsibility: The passage through childhood. Chicago: University of Chicago Press 
Henao, G., Ramírez, C. \& Ramírez, L. (2007). Las prácticas educativas familiares como facilitadoras del proceso de desarrollo en el niño y niña. El Ágora, 7 (2), 233-240.

Loreto, A., Pinilla, I. (2017). Enfoques metodológicos en la investigación educativa: Método empírico, cualitativo y fenomenológico. Recuperado de: http://www.guayanavirtual.web.ve/journaldata/journals/1/articles/7/public/7-103-2PB.pdf 